\title{
Variação sazonal nos atendimentos de emergência por asma em Gama, Distrito Federal*
}

\author{
Seasonal variations in emergency room visits for asthma attacks in Gama, Brazil
}

\author{
LAÉRCIO MOREIRA VALENÇA', PAULO CÉSAR NUNES RESTIVO², MÁRIO SÉRGIO NUNES³
}

\begin{abstract}
RESUMO
Objetivo: Quantificar o atendimento por asma em um serviço de emergência público e estudar a flutuação sazonal, considerando as condições climáticas locais, caracterizadas por duas estações, uma chuvosa e úmida e outra seca. Métodos: Levantamento retrospectivo conduzido em um hospital geral comunitário. Um total de 37.642 consultas emergenciais por asma, bronquite, pneumonia, infecções de vias aéreas superiores e outras condições foram identificadas durante um período de dois anos. As informações constantes em cada guia de atendimento foram levantadas para posterior análise. Resultados: Asma foi o segundo diagnóstico mais freqüente dentre as condições respiratórias (24\%). A maioria das consultas por asma foi feita em crianças de zero a catorze anos (56,6\%). A análise de regressão mostrou uma variação sazonal dos atendimentos, com concentração significativamente maior de casos de asma no mês de março ( $p=0,0109)$ e concentração menor nos meses de agosto e setembro ( $p=0,0485$ e $p=0,0169$, respectivamente). A correlação entre asma e clima foi mais significativa em relação à flutuação da umidade defasada de um e dois meses $(p=0,0026$ e $p=0,0002$, respectivamente). Conclusão: As visitas por asma ao pronto-socorro foram mais freqüentes durante a estação úmida. 0 aumento do número de casos cresceu um a dois meses após o aumento da umidade e diminuiu no período seco. Essa correlação positiva levanta a possibilidade de uma relação causal com a proliferação de ácaros domésticos e fungos ambientais.
\end{abstract}

Descritores: Estações do ano; Estado asmático/etiologia; Tempo (Meteorologia); Pyroglylphidae; Umidade; Serviço hospitalar de emergência

\begin{abstract}
Objective: To quantify the number of asthma attacks treated in the emergency room of a public hospital and to study seasonal fluctuations, taking into consideration the local climate, which is characterized by having only two seasons: a rainy/humid season and a dry season. Methods: A retrospective survey was conducted in a community general hospital. A total of 37,642 emergency room consultations related to asthma, bronchitis, pneumonia, upper-airway infection or other respiratory complaints were registered during a two year period. The data from each patient chart were collected for later analysis. Results: Among the respiratory conditions treated, asthma (24.4\%) was the second most common diagnosis. Most of the asthma consultations (56.6\%) involved children below the age of fifteen. Regression analysis revealed a seasonal variation in the number of asthma consultations, which was significantly higher in March $(p=0.0109)$, the low points being in August $(p=0.0485)$ and September $(p=0.0169)$. The correlation between climate and asthma was most significant in relation to changes in humidity, although the effect was delayed by one month $(p=0.0026)$ or two months ( $p=0.0002)$. Conclusion: Visits to the emergency room for the treatment of asthma attacks were more frequent during the rainy season, increasing at one to two months after the annual increase in humidity and decreasing in the dry season. This positive correlation raises the possibility of a causal relationship with proliferation of house dust mites and molds.
\end{abstract}

Keywords: Seasons; Status asthamaticus/etiology; Weather; Pyroglyphidae ; Humidity; Emergency service, hospital

\footnotetext{
* Trabalho realizado na Clínica Pneumológica do Hospital das Forças Armadas e na Universidade Católica de Brasília - UCB - Brasília (DF) Brasil. 1. Professor do Curso de Medicina da Universidade Católica de Brasília - UCB - Brasília (DF) Brasil.

2. Médico da Clínica Pneumológica do Hospital das Forças Armadas - HFA; Professor da Universidade Católica de Brasília - UCB - Brasília (DF) Brasil.

3. Médico da Secretaria da Saúde de Brasília - Brasília (DF) Brasil.

Endereço para correspondência: Laércio M. Valença. SMPW Qd. 16, Conj. 05, Casa 10 - CEP 71741-605, Brasília, DF, Brasil.

Tel: 5561 2107-5318. E-mail: Imvalenca@terra.com.br

Recebido para publicação em 9/6/05. Aprovado, após revisão, em 8/11/05.
} 


\section{INTRODUÇÃO}

Visitas à emergência e hospitalizações por asma decorrem de exacerbações da doença, constituem fatores de risco para morte e representam evidência de um controle terapêutico inadequado. Por isso, os guias práticos e consensos sobre o manejo da asma destacam, entre seus objetivos, a redução do número de consultas em pronto-socorro e de admissões hospitalares. ${ }^{(1)}$

0 agravamento sazonal da asma é um fenômeno reconhecido e vários estudos têm demonstrado uma variação nas taxas de atendimento emergencial, nas hospitalizações e na mortalidade em determinados períodos do ano. ${ }^{(2-3)} 0$ estudo dos padrões de utilização do pronto-socorro por asmáticos pode ser um melhor indicador que a análise de hospitalizações, pois compreende uma população maior de pacientes e com um amplo espectro de gravidade. A identificação de uma periodicidade sazonal pode prover elementos para a pesquisa de fatores ambientais e de respostas orgânicas alteradas que subsidiem a instituição de medidas preventivas.

0 objetivo do presente levantamento foi quantificar o atendimento por asma em um serviço de emergência público e estudar sua variação sazonal, considerando as condições climáticas locais, caracterizadas por duas estações distintas, uma chuvosa e úmida e outra seca.

\section{MÉTODOS}

Este estudo foi conduzido em um hospital geral comunitário com 457 leitos, responsável por 98\% dos leitos da cidade satélite do Gama (DF). Esse núcleo populacional dista $35 \mathrm{~km}$ do centro de Brasília (DF) e contava com uma população de 133.797 habitantes, com uma renda per capita de R\$3.427,82 (US\$ 1,874,00), no ano 2000. 0 clima, com uma precipitação anual média de 1.244,8 mm, é caracterizado por uma estação chuvosa de outubro a abril e uma estação seca de maio a setembro. A temperatura diminui de maio a agosto, atingindo uma mínima de $14,6^{\circ} \mathrm{C}$, em média.

Neste levantamento, os dados foram coletados retrospectivamente de $1^{\circ}$ de janeiro de 1999 a 31 de dezembro de 2000. Ao longo desses dois anos houve 641.905 consultas no serviço de pronto-socorro do hospital. Do total, 37.642 consultas foram realizadas em virtude de queixas respiratórias. As informações contidas em cada uma das guias de atendimento de emergência, tais como iniciais do nome, data de nascimento, sexo, residência, data e hora da entrada, diagnóstico e destino (alta da emergência ou internação), foram digitadas em uma base de dados Epi-Info para subseqüente análise. Os diagnósticos foram transcritos na forma registrada pelos médicos de plantão e agrupados de acordo com o Código Internacional de Doenças CID -10: asma (J45, J46); bronquite aguda (J20); pneumonia (J12-J18); infecção do trato respiratório superior (J00-J06 e J11, exceto tonsilite aguda); outros diagnósticos. Não foi feita tentativa de validação dos diagnósticos firmados pelos médicos. Os dados climáticos foram obtidos do Instituto Nacional de Meteorologia. Neste trabalho, os dados foram condensados como médias anuais ou mensais.

Em relação à análise estatística, foi utilizado o coeficiente de correlação linear de Pearson para se avaliar a correlação entre diversas variáveis, calculando-se os respectivos valores de $\mathrm{p}$ e intervalos de confiança. Para se estudar a variação sazonal, foi inicialmente aplicada a transformação logarítmica à média mensal do número de atendimentos por asma nos anos de 1999 e 2000, estudados conjuntamente, para tornar a série mais homogênea. A seguir, foi empregado um modelo de regressão linear múltiplo com doze variáveis independentes, correspondentes aos meses de janeiro a dezembro. ${ }^{(4)}$ Foi igualmente pesquisada a oscilação sazonal do total de atendimentos feitos no pronto-socorro, de forma a se excluir que a variação observada fosse decorrente de variações nos atendimentos totais, usando-se o programa estatístico SAS, versão 8.2.

\section{RESULTADOS}

A média e o percentual anuais das condições respiratórias atendidas no serviço de emergência são mostrados na Tabela 1. Asma foi o segundo diagnóstico mais freqüente $(24,4 \%)$, só ficando atrás das infecções do trato respiratório superior $(47,1 \%)$.

Dos pacientes com asma, 51,3\% eram do sexo masculino. A maioria das consultas por asma foi feita em crianças de zero a catorze anos $(52,6 \%)$, predominando na faixa de zero a quatro anos $(29,4 \%)$ e de cinco a nove anos $(16,7 \%)$. A taxa anual de atendimentos por asma foi de 343 visitas/ 10.000 habitantes. Os grupos etários mais baixos 
TABELA 1

Média e percentual anuais das condições respiratórias atendidas no serviço de emergência

\begin{tabular}{lcc}
\hline Diagnóstico & Média anual & Percentual anual \\
\hline Infecção do trato & & \\
respiratório superior & 8.879 & 47,1 \\
Asma & 4.591 & 24,4 \\
Bronquite aguda & 2.780 & 14,6 \\
Pneumonia & 1.976 & 10,4 \\
DPOC & 230 & 1,2 \\
Bronquiolite & 164 & 0,9 \\
Outros diagnósticos & 301 & 1,6 \\
\hline Total & 18.821 & 100,0 \\
\hline
\end{tabular}

DP0C: doença pulmonar obstrutiva crônica

contribuíram com taxas ainda maiores (977 para zero a quatro anos e 661 para cinco a nove anos por 10.000 habitantes do respectivo grupo etário).

0 maior número de consultas por asma ocorreu nos meses de janeiro a março $(39,7 \%)$, enquanto que o menor número teve lugar no trimestre de julho a setembro (14,7\%). A análise de regressão mostrou concentrações significativamente maiores de casos de asma no mês de março e concentrações significativamente menores nos meses de agosto e setembro $(p=0,0109, p=0,0485$ e $p$ $=0,0169$, respectivamente), caracterizando uma variação sazonal.

Os resultados da análise de regressão do nú- mero total de casos atendidos no pronto-socorro, incluindo todas as especialidades, mostraram também uma variação sazonal, mas diferente da distribuição da asma, com maior número de casos em maio e junho ( $p=0,0011$ e 0,0263, respectivamente) e menores concentrações em julho e setembro ( $p=0,076$ e 0,0047 , respectivamente). Não houve correlação entre número total de atendimentos e visitas por asma.

A precipitação pluviométrica nos trimestres de janeiro a março e de julho a setembro foi 467,8 $\mathrm{mm}$ e $113,5 \mathrm{~mm}$, respectivamente, e a umidade relativa de $67,7 \%$ e $44,7 \%$, respectivamente. Contudo, não houve correlação importante entre atendimentos por asma e variáveis climáticas (umidade, precipitação pluviométrica e temperatura média), de acordo com a análise estatística apresentada na Tabela 2. Mas como o número de visitas por asma começou a subir um a dois meses após a elevação da umidade, conforme visto na Figura 1, estudou-se a correlação entre asma e a umidade relativa do ar defasada de um e de dois meses, encontrando-se uma correlação linear positiva e estatisticamente significativa, ou seja, quanto maior a umidade relativa do ar nos dois meses anteriores maior foi o número de atendimentos por asma. Encontrou-se também uma correlação positiva e significativa entre precipitação de chuva e temperatura média defasadas de dois meses em relação às visitas emergenciais por asma (Tabela 2).

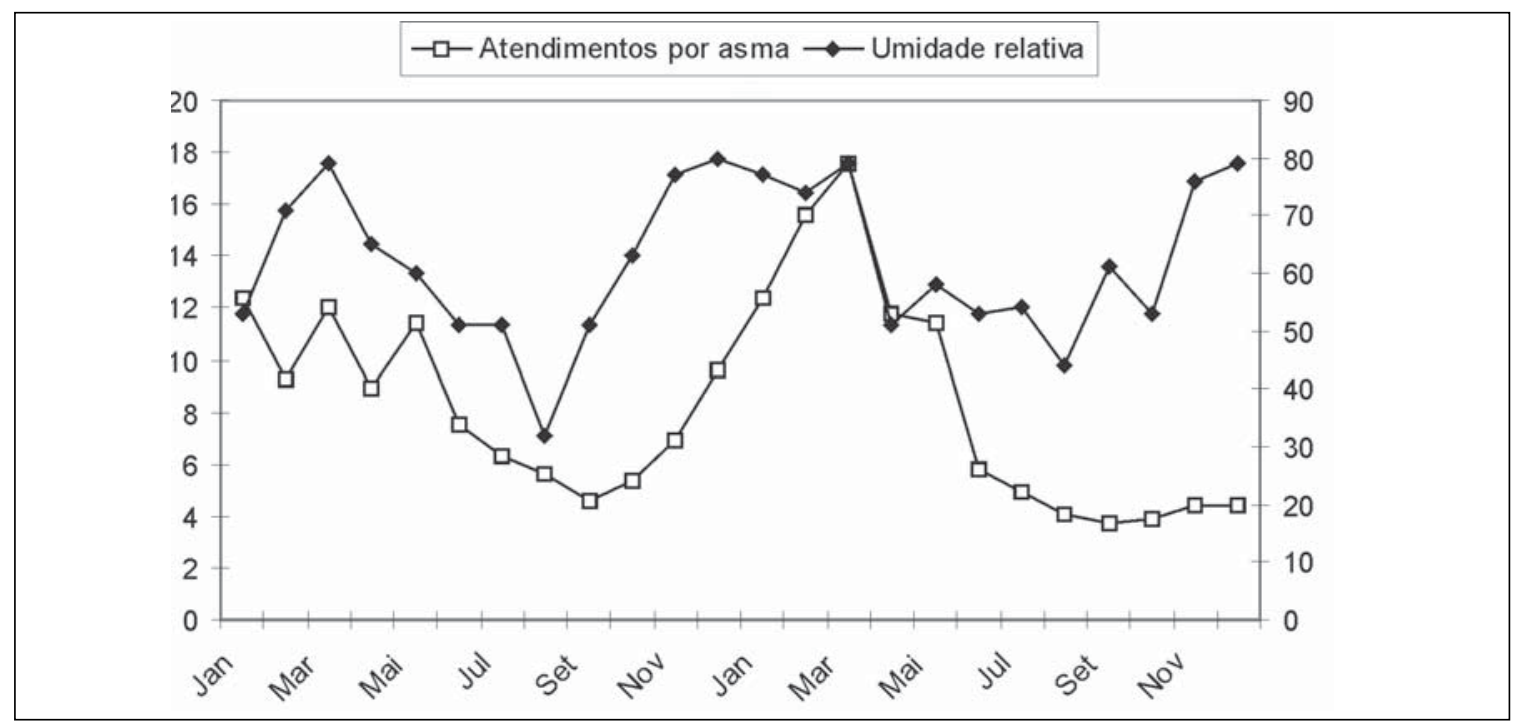

Figura 1 - Variação percentual dos atendimentos por asma no serviço de emergência e da umidade relativa do ar nos anos de 1999 e 2000 
TABELA 2

Correlação ao longo dos anos de 1999 e 2000 entre atendimentos por asma, variáveis meteorológicas e outras condições respiratórias vistas no pronto-socorro

\begin{tabular}{lcrc}
\hline Atendimentos por asma versus & $\mathrm{r}$ & Intervalo de confiança $95 \%$ & $\mathrm{p}$ \\
\hline Umidade relativa do ar & 0,373 & $-0,036-0,675$ & 0,0725 \\
Umidade relativa do ar defasada de um mês & 0,587 & $0,231-0,805$ & 0,0026 \\
Umidade relativa do ar defasada de dois meses & 0,690 & $0,378-0,861$ & 0,0002 \\
Precipitação pluviométrica & 0,140 & $-0,279-0,514$ & 0,0725 \\
Precipitação pluviométrica defasada de um mês & 0,344 & $-0,079-0,662$ & 0,1085 \\
Precipitação pluviométrica defasada de dois meses & 0,573 & $0,200-0,801$ & 0,0045 \\
Temperatura média & 0,128 & $-0,291-0,505$ & 0,5558 \\
Temperatura média defasada de um mês & 0,369 & $-0,051-0,678$ & 0,0835 \\
Temperatura média defasada de dois meses & 0,428 & $0,008-0,720$ & 0,0462 \\
\hline Total de consultas no serviço de pronto-socorro & 0,248 & $-0,172-0,592$ & 0,2452 \\
\hline
\end{tabular}

r: coeficiente de correlação

\section{DISCUSSÃO}

0 presente estudo registra pela primeira vez na literatura médica brasileira, com base em pesquisa bibliográfica realizada no LILACS e Scielo, que os atendimentos por asma no pronto-socorro, abrangendo crianças e adultos, apresentaram uma variação sazonal, com um significativo aumento de casos em março e um nadir nos meses de agosto e setembro. Também ficou evidenciado que os casos agudos de asma aumentaram de um a dois meses após a elevação da umidade relativa do ar e dois meses após o aumento do índice pluviométrico e da temperatura média. A defasagem entre mudanças nas condições ambientais e crises de asma sugere que o intervalo seria o tempo requerido para ocorrer um aumento na concentração de possiveis fatores desencadeantes, tais como ácaros e/ou fungos.

A validade desses achados poderia ser questionada por limitações inerentes à utilização de dados diagnósticos firmados por equipes médicas de pronto-socorro. Contudo, eventuais impropriedades diagnósticas, inclusive nos grupos etários mais jovens, são atenuadas pela facilidade maior em se diagnosticar crise de asma do que asma em remissão, e por uma alta média anual de 4.591 atendimentos. Metodologia similar foi usada por outros autores, inclusive na faixa etária infantil. . $^{(3,5-7)}$

Flutuações na morbidade por asma têm sido observadas em outros países. Alguns autores ${ }^{(5)}$ verificaram, no Canadá, um pico de atendimento emergencial por asma começando na última sema- na de setembro e continuando por três semanas. Este pico afetou crianças e adultos com idades entre 15 e 60 anos, mas nenhum aumento foi visto entre os indivíduos acima de 60 anos. Não foi encontrada uma correlação entre o aumento nas visitas por asma e níveis de dióxido de nitrogênio e dióxido de enxofre. Pareceu improvável a esses pesquisadores que um pólen específico ou a replicação de ácaros fossem os responsáveis pelo evento. A causa do pico sazonal não foi, portanto, identificada.

Nos EUA também tem sido encontrado um maior número de visitas à emergência no outono e um menor número no verão. ${ }^{(6,8)}$ Alguns autores ${ }^{(8)}$ verificaram que os padrões sazonais foram similares entre crianças sem reatividade ao teste cutâneo, aqueles com reatividade somente aos alérgenos domiciliares e aqueles reativos aos alérgenos ambientais externos. Entre os poluentes avaliados, somente o padrão sazonal do dióxido de enxofre coincidiu com o da morbidade por asma, mas uma revisão da literatura lançou dúvidas sobre a natureza causal dessa associação. Outros autores ${ }^{(7)}$ observaram flutuações sazonais maiores em crianças abaixo dos catorze anos.

Assim, nos trabalhos publicados até o presente, não foram identificadas causas para a variação sazonal dos ataques de asma. Dentre os possíveis fatores envolvidos, uma associação entre infecções respiratórias virais e crises de asma foi documentada em vários estudos..$^{(9-12)} \mathrm{Um}$ estudo ${ }^{(11)}$ deu suporte à hipótese de que infecções virais do trato respiratório alto estão associadas com $80 \%$ a $85 \%$ 
das exacerbações por asma em crianças em idade escolar. Já em uma pesquisa em crianças, no Brasil, o rinovírus não pareceu ser um desencadeante de exacerbações asmáticas. ${ }^{(13)}$

Tem-se investigado a relação entre a presença de fungos no ambiente e asma. 0 crescimento de mofo na residência pode levar a doença respiratória grave, requerendo hospitalização. ${ }^{(14)}$ Alguns autores ${ }^{(15-16)}$ observaram correlação entre esporos de fungos e exacerbações asmáticas. Um outro estudo mostrou que dez entre onze pacientes com asma que tiveram parada respiratória tinham teste cutâneo positivo para Alternaria alternata, em comparação com 31\% dos indivíduos controle. ${ }^{(17)} \mathrm{Em}$ um estudo ${ }^{(18)} \mathrm{em}$ indivíduos com idades de cinco a 34 anos, observou-se que mortes relacionadas a asma eram mais prováveis de ocorrer em dias com contagem de esporos mais alta $(=1.000$ esporos por $\mathrm{m}^{3}$ ). Concluiu-se que, embora mortes causadas por asma também envolvam fatores pessoais, sociais e de acesso a serviços médicos, os achados do estudo sugeriam que a exposição a fungos ambientais pudesse ter um papel na mortalidade por asma e deveria ser considerada em estratégias preventivas.

Em um levantamento realizado no Distrito Federal, ${ }^{(19)}$ verificou-se que, entre 1.376 indivíduos examinados em um serviço de pneumologia, 26\% tinham um teste cutâneo por puntura positivo para alérgenos. Destes, 63\% reagiram a antígenos de ácaros, $22 \%$ e $19 \%$ a misturas diferentes de fungos e $11 \%$ tanto a pêlo de gato como de cão.

Acredita-se há muito tempo que alérgenos de ácaros desempenham um papel importante na provocação de sintomas asmáticos. ${ }^{(20)} \mathrm{Na}$ cidade de Wellington, Nova Zelândia, 76\% dos asmáticos que tinham sido hospitalizados recentemente tiveram teste cutâneo positivo para $D$. pteronyssinus. ${ }^{(21)}$ Motivados por esses achados, alguns autores ${ }^{(22)}$ verificaram níveis de Der 1 significativamente mais altos em casas com carpetes velhos do que naquelas com novos ou sem carpetes, e com umidade relativa acima da média (51\%). Outros ${ }^{(23)}$ examinaram a poeira de colchão coletada em intervalos mensais por dois anos e meio. Ácaros vivos foram encontrados nos meses mais quentes e quando a umidade relativa era superior a 50\% por pelo menos parte de cada dia durante o mês de coleta. Embora o pico da população de ácaros tenha variado de um ano para outro, os dados gerais apontaram no sentido de que a umidade e a temperatura mais altas favorecem a proliferação de ácaros. No período englobado pelo presente estudo, a umidade relativa do ar no Distrito Federal foi, em média, de $67,7 \%$ no trimestre de janeiro a março, e de $47,7 \%$ no trimestre de julho a setembro.

0 presente estudo identificou outros dados de interesse médico, como o fato de que a asma é responsável por um quarto das emergências respiratórias em um hospital comunitário no centro-oeste do Brasil. Este valor ficou abaixo dos 30\% na cidade de São Paulo (SP) ${ }^{(24)}$ e dos 41,3\% encontrados em Vancouver, Canadá. ${ }^{(5)}$ Quando relacionada à população, a taxa anual de visitas ao pronto-socorro neste estudo foi de 343/10.000, portanto quase dez vezes maior que a cifra verificada em Vancouver (35/10.000). Pouco mais da metade das consultas emergenciais por asma foram feitas em crianças entre zero e catorze anos de idade e quanto mais jovem o grupo etário maior foi a taxa de visitas (977/10.000 no grupo de zero a quatro anos e 661/10.000 no de cinco a nove anos). Estas taxas são 2,8 e 1,9 vezes mais elevadas que a da população geral. A taxa no grupo de zero a quatro anos foi apenas um pouco menor que a encontrada em um estudo em crianças seguradas pelo Medicaid, nos EUA (1.074/10.000 crianças seguradas). ${ }^{(6)}$ Estes números indicam que a morbidade por asma é maior em crianças e que grupos sociais menos privilegiados recorrem mais à emergência por não terem acesso a serviços ambulatoriais e a um tratamento preventivo adequado.

Em conclusão, os nossos dados mostram que as visitas emergenciais por asma sofrem variação sazonal, crescem um a dois meses após o aumento da umidade relativa do ar e caem no período mais seco do ano. É reconhecido que a presença de inflamação e de hiper-responsividade brônquica tornam as vias aéreas suscetíveis a diversos agentes específicos (alérgenos) e não específicos (infecções virais, ar frio, exercício, fumo passivo e agentes farmacológicos) que podem causar obstrução das vias aéreas e sintomas asmáticos. Assim, é plausível a hipótese de que o desencadeamento de crises de asma durante a estação úmida e mais quente, em nossa região, seja favorecido particularmente pela proliferação de ácaros e fungos. Nesse caso, a adoção de medidas preventivas e de controle ambiental pode contribuir para reduzir a morbidade por asma. 


\section{AGRADECIMENTOS}

Agradecemos ao Prof. Dr. Paulo Sérgio Beraldo Siebra pela revisão do trabalho e construtivas sugestões apresentadas.

\section{REFERÊNCIAS}

1. Sociedade Brasileira de Pneumologia e Tisiologia. 1 Consenso brasileiro no manejo da asma. J Pneumol. 1998;24(4):171-276.

2. Weiss KB. Seasonal trends in US asthma hospitalizations and mortality. JAMA. 1990;263(17):2323-8.

3. Fleming DM, Cross KW, Sunderland R, Ross AM. Comparison of the seasonal patterns of asthma identified in general practitioner episodes, hospital admissions, and deaths. Thorax. 2000;55(8):662-5.

4. Neter J, Wasserman W, Kutner MH. Applied linear regression models (Richard D. Irwin, Inc., Illinois, 1983)

5. Bates DV, Baker-Anderson M, Sizto R. Asthma attack periodicity: a study of hospital emergency visits in Vancouver. Environ Res. 1990;51(1):51-70.

6. Fredrickson DD, Molgaard CA, Dismuke SE, Schukman JS, Walling A. Understanding frequent emergency room use by Medicaid-insured children with asthma: a combined quantitative and qualitative study. J Am Board Fam Pract. 2004;17(2):96-100.

7. Silverman RA, Stevenson L, Hastings HM. Age-related seasonal patterns of emergency department visits for acute asthma in an urban environment. Ann Emerg Med. 2003;42(4):577-86.

8. Gergen PJ, Mitchell H, Lynn H. Understanding the seasonal pattern of childhood asthma: results from the National Cooperative Inner-City Asthma Study (NCICAS). J Pediatr. 2002;141(5):631-6. Comment in: J Pediatr. 2002;141(5):604-5.

9. Nicholson KG, Kent J, Ireland DC. Respiratory viruses and exacerbations of asthma in adults. BMJ. 1993;307(6910): 982-6. Comment in: BMJ. 1994;308(6920):57.

10. Gern JE, Busse WW. Association of rhinovirus infections with asthma. Clin Microbiol Rev. 1999;12(1):9-18.

11. Johnston SL, Pattemore PK, Sanderson G, Smith S, Lampe F, Josephs L, et al. Community study of role of viral infections in exacerbations of asthma in 9-11 year old children. BMJ. 1995;310(6989):1225-9. Comment in: BMJ. 1995;311(7005):629. BMJ. 1995;311(7005):629-30.

12. Fraenkel DJ, Bardin PG, Sanderson G, Lampe F, Johnston
SL, Holgate ST. Lower airways inflammation during rhinovirus colds in normal and in asthmatic subjects. Am J Respir Crit Care Med. 1995;151(3 Pt 1):879-86.

13. Câmara AA, Silva JM, Ferriani VP, Tobias KR, Macedo IS, Padovani MA, et al. Risk factors for wheezing in a subtropical environment: role of respiratory viruses and allergen sensitization. J Allergy Clin Immunol 2004;113(3):551-7.

14. Solomon WR. Fungus aerosols arising from cold-mist vaporizers. J Allergy Clin Immunol. 1974;54(4):222-8.

15. Salvaggio J, Seabury J, Schoenhardt FA. New Orleans asthma. V. Relationship between Charity Hospital asthma admission rates, semiquantitative pollen and fungal spore counts, and total particulate aerometric sampling data. J Allergy Clin Immunol. 1971;48(2):96-114.

16. Dales RE, Cakmak S, Burnett RT, Judek S, Coates F, Brook JR. Influence of ambient fungal spores on emergency visits for asthma to a regional children's hospital. Am J Respir Crit Care Med. 2000;162(6):2087-90.

17. O'Hollaren MT, Yunginger JW, Offord KP, Somers MJ, O'Connell EJ, Ballard DJ, et al. Exposure to an aeroallergen as a possible precipitating factor in respiratory arrest in young patients with asthma. $\mathrm{N}$ Engl $\mathrm{J}$ Med. 1991;324(6):359-63. Comment in: N Engl J Med. 1991;324(6):409-11. N Engl J Med. 1991;325(3):206-8.

18. Targonski PV, Persky VW, Ramekrishnan V. Effect of environmental molds on risk of death from asthma during the pollen season. J Allergy Clin Immunol. 1995;95(5 Pt 1):955-61.

19. Pereira AAF, Valença LM, Restivo PCN. Testes cutâneos alérgicos em doentes respiratórios. Brasília Med. 1997;34 Supl 1:92.

20. Peat JK, Tovey E, Toelle BG, Haby MM, Gray EJ, Mahmic A, et al. House dust mite allergens. A major risk factor for childhood asthma in Australia. Am J Respir Crit Care Med. 1996;153(1):141-6.

21. Fitzharris P, Stone L, Sawyer G. The atopic profile of adult asthmatics admitted to hospital in Wellington, New Zealand [abstract]. J Allergy Clin Immunol. 1996;97:378

22. Wickens R, Siebers R, Ellis I, Lewis S, Sawyer G, Tohill S, et al. Determinants of house dust mite allergen in homes in Wellington, New Zealand. Clin Exp Allergy. 1997;27(9): 1077-85.

23. Murray AB, Zuk P. The seasonal variation in a population of house dust mites in a North American city. J Allergy Clin lmmunol. 1979;64(4):266- 9.

24. Oliveira MA, Bruno VF, Ballini LS, Brito Jardim JR, Fernandes AL. Evaluation of an educational program for asthma control in adults. J Asthma. 1997;34(5):395-403. 